\title{
Cultura ciudadana y convivencia escolar desde el uso de la investigación como estrategia pedagógica, apoyada en las tecnologías de la información y la comunicación ${ }^{1}$ \\ Citizen culture and student coexistence from the use of research as a pedagogical strategy supported by Information and communication technologies.
}

DOI: http://dx.doi.org/10.17981/cultedusoc.9.3.2018.0

Artículo de investigación. Fecha de recepción: 15/06/2018. Fecha de aceptación: 27/11/2018

\author{
Adela Borja Orozcón; \\ Aura Caballero; Rita Pertuz Ortiz; María Romero Ortiz; Manuel Rodríguez Vega; \\ Yanelis Lidueñas; Cristóbal García; Luis Gutiérrez Ceballos; \\ Nilson Ortiz Torregroza; Hugo Orozco Gutiérrez; Guadalupe Ortiz Pozo; \\ Matías Vargas Morales; Ena Orozco Orozco; Josman Álvarez Ternera; \\ Luz Mila Mercado; Nancy De La Hoz y Saime Pertuz Orozco ${ }^{3}$ \\ Institución Educativa Agropecuaria Otilia Mena Álvarez (Colombia) \\ adelaborjaorozco@gmail.com
}

Para citar este artículo:

Borja, A., Caballero, A., Pertuz, R., Romero, M., Rodríguez, M., Lidueñas, Y., García, C., Gutiérrez, L., Ortiz, N., Orozco, H., Ortiz, G., Vargas, M., Orozco, E., Álvarez, J., Mercado, L., De La Hoz, N. y Pertuz, S. (2018). Cultura ciudadana y convivencia escolar desde el uso de la investigación como estrategia pedagógica, apoyada en las tecnologías de la información y la comunicación. Cultura. Educación y Sociedad 9(3), 85-90. DOI: http://dx.doi.org/10.17981/cultedusoc.9.3.2018.0

\section{Resumen}

La integración al currículo de la investigación como estrategia pedagógica (IEP) y el uso de las tecnologías de la información y la comunicación (TIC), tiene como objetivo generar propuestas novedosas a partir de las cuales se propone impactar a la comunidad estudiantil en el desarrollo de la cultura ciudadana y el mejoramiento de la convivencia escolar. El propósito de la presente investigación es generar cultura ciudadana y convivencia estudiantil desde el uso de la IEP apoyada en las TIC. El estudio es de tipo cualitativo. La población estuvo conformada por 190 estudiantes de la Institución Educativa Agropecuaria Otilia Mena Álvarez. Para la recolección de datos se realizó un diagnóstico apoyado en las TIC de la cultura ciudadana y convivencia escolar en la institución. A partir de la investigación, como estrategia pedagógica se logró fomentar la reflexión crítica de los estudiantes frente a la problemática de convivencia escolar y cultura ciudadana resaltando la tolerancia y buenas relaciones personales.

Palabras clave: Cultura ciudadana y convivencia escolar, Investigación como estrategia pedagógica, Tecnología de información y comunicación

\section{Abstract}

The integration into the curriculum of research as a pedagogical strategy (IEP) and the use of information and communication technologies (ICT) generates novel proposals from which it is proposed to impact the student community in the development of citizen culture and the improvement of school coexistence. The purpose of this research is to generate citizen culture and student coexistence from the use of the IEP supported by ICT. The study is of a qualitative, proactive type. The population consisted of 190 students of the Otilia Mena Alvarez Agricultural Educational Institution. For data collection, a diagnosis was made based on the ICT of the citizen culture and school coexistence in the institution, and work tables and workshops of contextualized experiences were developed with students, teachers and parents. From the research, as a pedagogical strategy it was possible to foster the critical reflection of the students in the face of the problem of school coexistence and civic culture highlighting tolerance, good personal relationships and respect for individual differences.

Keywords: Citizen culture, School life, Research as a pedagogical strategy, Information and communication technology.

1 Este artículo ha sido derivado del Programa de Fortalecimiento de la Cultura Ciudadana y Democrática CT+I a través de la IEP apoyada en TIC en el Departamento de Magdalena: CICLON

2 Líder del grupo de investigación: "Aventureros del saber".

3 Docentes de la Institución Educativa Agropecuaria Otilia Mena Álvarez.

- The author; licensee Universidad de la Costa - CUC

Cultura, Educación y Sociedad vol. 9 no. 3, pp. 85-90. Diciembre, 2018

Barranquilla. ISSN 2389-7724 Online 


\section{Introducción}

La integración al currículo de la investigación como estrategia pedagógica (IEP) y el uso de las tecnologías de la información y comunicación (TIC), genera propuestas novedosas a partir de las cuales se propone impactar a la comunidad estudiantil en el desarrollo de la cultura ciudadana y el mejoramiento de la convivencia escolar, teniendo en cuenta que, Valenzuela y Rochin ( 2013) indican que "los medios de comunicación en diversas ocasiones tienen intereses vinculados con grupos de poder, actores políticos y el mismo gobierno; por lo mismo, en calidad de aparatos ideológicos mantienen el status quo informando lo que el sistema quiere" (p. 91).

La Institución Educativa Agropecuaria Otilia Mena Álvarez, se ubica en un área rural y cuenta con 469 estudiantes, pese a no contar con una amplia cantidad de estudiantes, en ella convergen una serie de dificultades a nivel de convivencia escolar siendo que, tanto en la primaria como en el bachillerato, el ambiente es conflictivo. Existe la impresión que muchas de las debilidades identificadas se acentúan en poblaciones de gran vulnerabilidad. Al respecto, Romero y Contreras (2015), destacan como factores clave para la participación y el empoderamiento social, las condiciones de vulnerabilidad y postconflicto que han transitado muchas comunidades en las regiones de Colombia. En este amrco referencial y tomando en consideración la complejidad social multifactorial, así como la vulnerabilidad, condiciones económicas y familias disfuncionales. se gestó el equipo de investigación llamados: "Aventureros del saber", conformado por un grupo de docentes, a través del cual se realizó una propuesta frente a la creciente demanda de generar estrategias para mitigar en la comunidad estudiantil la aparición de conductas que dificultan el debido proceso de convivencia, además de las notorias falencias en la formación de cultura ciudadana.
La necesidad de responder a las dificultades anteriormente mencionadas no se refleja en el trabajo institucional para generar cambios en cuanto a la convivencia escolar; a esto se suma la responsabilidad de las instituciones educativas en formar ciudadanos capaces de convivir y con cultura ciudadana, para contrarrestar las múltiples problemáticas existentes en la sociedad actual.

A partir de lo expuesto en el Plan Nacional Decenal de Educación 2006-2016, los principales objetivos de la educación en Colombia, se encuentran dirigidos a la promoción de una educación focalizada en fomentar la sana convivencia y la ciudadanía a través del diseño y la aplicación de programas que garanticen un desarrollo integral en el marco del contexto escolar.

De acuerdo con Bolívar (2004), la educación pública se constituye en la base para la formación de la cultura ciudadana, desde la implementación de diversas alternativas que permitan la integralidad de opciones, apostando a la promoción y formación de espacios que posibiliten la convivencia en un marco común.

Por su parte (Belalcazar y Molina, 2017), destacan la importancia existente frente al rol docente y su capacidad de identificar las múltiples dificultades de convivencia que afrontan las entidades educativas en la actualidad, con la finalidad de proponer novedosas estrategias de intervención que conlleven al planteamiento de diversas alternativas de solución acordes con la contemporaneidad, que den respuestas en pro a la sana convivencia a partir de la práctica educativa, incorporando procesos de sistematización que involucren los recursos de las TIC.

Teniendo en cuenta lo propuesto por (Chaux, Lleras, y Velásquez 2004; citado por Vargas, 2016), es pertinente aludir que, en un área de aprendizaje que incluye las TIC y requiere formar a la comunidad, se fomenta el aprendizaje activo, independiente y colaborativo, haciendo un llamado 
al estudiante, a enfrentar situaciones variantes abordadas desde un pensamiento crítico.

La cultura ciudadana, comprende un trasfondo de sentido que regula los comportamientos de los ciudadanos, en la medida que establece las reglas mínimas que hacen posible las relaciones sociales del ser humano con su entorno (Illera, 2005). En este mismo sentido, Samper y Maussa (2014) sostienen que las competencias ciudadanas de los sujetos del nuevo milenio han de priorizar el cuidado de la vida, el cuidado del planeta y la protección del medio ambiente.

Cuando se habla de convivencia, de acuerdo a Marrugo et al. (2016), se alude a la vida compartida entre las personas, familias y grupos en cuanto a intereses, problemas, inquietudes; en fin, todo aquello que forma parte de la vida en sociedad. En este sentido, la convivencia escolar alude al compartir de manera pacífica e integrada el contexto educativo, y en la cual media los procesos de aprendizaje y el desarrollo integral de los estudiantes (Mena, Romagnoli, y Valdés, 2006).

La sana convivencia es un elemento vital en los procesos educativos, en todos sus niveles, en tanto que permite que las relaciones se desarrollen de manera constructiva, siendo importante aclarar, que la convivencia no implica la ausencia de conflictos (Ruiz y Chaux, 2005), sino la resolución adecuada de estos. Así mismo, siendo esta parte del proceso educativo, implica que todos los miembros de la comunidad escolar tienen responsabilidad, en la manera de interrelacionarse y en los procesos de convivencia (Megías, 2011).

De acuerdo a Castro-Alfaro et al. (2015), las instituciones educativas deben esforzarse por generar estrategias que busquen de manera constante la mejora en la convivencia entre los estudiantes que están formando, a través de valores como el respeto y la tolerancia.
En relación a lo anterior, Garrell (2009), explica la pertinencia de fomentar un clima favorable en la escuela y buscar diferentes alternativas de solución para invitar a los estudiantes a reflexionar sobre sus ideas y comportamientos, propiciando así una convivencia en armonía.

En cuanto al uso de las TIC, en el desarrollo de las estrategias utilizadas para alcanzar el objetivo planteado, son herramientas tecnológicas que permiten el desarrollo de procesos innovadores que pueden ser manejados en cualquier campo y aplicados en la práctica pedagógica para fortalecer procesos de aprendizaje significativos (Ramírez, 2010). En relación a esto, se plantea que las TIC han adquirido cada vez más relevancia en las prácticas educativas para contribuir al proceso de formación integral de los estudiantes.

La investigación como estrategia pedagógica (IEP), permite la promoción de espacios para que los diferentes actores incorporen el uso de tecnologías de la información y las comunicaciones (TIC), para potenciar el acompañamiento y las actividades investigativas, formarse colaborativamente e individualmente, dar a conocer sus trabajos, compartir conocimientos, y reflexionar sobre las experiencias de virtualidad, con el fin de construir una cultura de uso de esos recursos tecnológicos en los diferentes procesos pedagógicos e investigativos (Colciencias, 2011).

En función de lo planteado, surge la iniciativa de integrar la IEP al currículo institucional, teniendo en cuenta que esta posibilita la puesta en escena de otros recursos, en este caso tecnológicos, que orientaran los procesos académicos y reflexivos.

En palabras de Mejía y Manjarrez (2010), la IEP reconoce la investigación como una forma de cambiar concepciones, realidades y el entorno de los participantes, y con ello, una manera de situarse crítica y éticamente en el mundo cercano, mediato y global. Es por tanto la mejor forma de 
fomentar la criticidad e investigación generando a través de temas pertinentes, $\mathrm{y}$ del interés mismo de los estudiantes, una estrategia que los eduque y a la vez impacte positivamente en las relaciones que se gesten por la realización de trabajo en equipo.

Finalmente, en el mundo actual resulta indispensable dinamizar los espacios educativos por medio de herramientas tecnológicas que se convierten en un aliado para los docentes. En este caso, se utilizó para el desarrollo de estrategias investigativas y recreativas que decantan en la apropiación de conocimientos y reflexiones que, a su vez, resultaron en una mejor apropiación de la cultura ciudadana y en la transversalidad del proyecto en el impulso de mejores relaciones de convivencia dentro del marco institucional.

Entendiendo el ejercicio de la ciudadanía en la actualidad directamente relacionada con las tecnologías de información y comunicación (TICs), donde el ciudadano ejecute acciones y manifieste actitudes como miembro de una comunidad en los asuntos de interés común, impulsando la interrelación entre organizaciones sociales y ciudadanas, incluyendo las educativas (Hernández, Peña y Velazco; 2013).

\section{Metodología}

La presente investigación se desarrolla desde un enfoque metodológico cualitativo, el cual "se enfoca a comprender y profundizar los fenómenos, explorándolos desde la perspectiva de los participantes en un ambiente natural y en relación con el contexto" (Hernández, Collado \& Baptista, 2010). Así mismo, se utilizó un modelo de tipo propositivo, que alude a un proceso conjunto entre los distintos participantes de la investigación, con el fin de fomentar la reflexión y generar estrategias para dar solución a un problema en específico. Con este tipo de metodología se buscó generar cultura ciudadana y convivencia estudiantil, a partir de la reflexión crítica de los estudiantes y sobre conductas relacionadas a estas que dificultan los procesos educativos y la sana convivencia, considerando experiencias contextualizadas a su entorno y realidad.

Se seleccionó una población que corresponde a estudiantes de la Institución Educativa Agropecuaria Otilia Mena Álvarez, la cual se divide en dos sedes, primaria y bachillerato, con un total de 469 estudiantes, de los cuales se utilizó una muestra de 190, de los cursos tercero, quinto, sexto, octavo y décimo.

Para el proceso de recolección de información se realizó la aplicación virtual, mediante el uso de tablets, de un instrumento de evaluación de la convivencia escolar, tanto a estudiantes como a docentes, con el fin de realizar un diagnóstico de la misma. De igual forma, se realizaron talleres con los padres de familia para evidenciar los procesos de convivencia a nivel familiar. Finalmente, se desarrollaron mesas de trabajo y talleres de experiencias contextualizadas con los estudiantes, a través de lecturas y videos, para la reflexión crítica en torno a valores como la tolerancia, el respeto por las diferencias, y la convivencia, así como la construcción de huertos escolares, cuyo propósito fue fomentar el trabajo colaborativo y cuidado por el bien común.

\section{Resultados}

El estudio desarrollado con estudiantes de primaria y bachillerato de la Institución Educativa Agropecuaria Otilia Mena Álvarez, que se desarrolló a partir de la IEP mediante la utilización de las TIC, promovió espacios de interacción en los distintos niveles de la comunidad educativa (estudiantes, padres y docentes) y permitió evidenciar cómo la mala convivencia y poca cultura ciudadana responden a aspectos multifactoriales que dan cuenta de la complejidad social del entorno del estudiante. 
Durante la experiencia significativa que buscó fomentar desde la IEP un aprendizaje colaborativo, contextualizado y problematizador, los estudiantes mostraron receptividad frente a las temáticas abordadas en las distintas actividades, evidenciándose una inquietud y razonamiento frente a múltiples situaciones de convivencia y tolerancia que se propician en la escuela, permitiendo que plasmaran sus reflexiones en relatos escritos en los cuales describieron sus perspectivas y respuestas ante las preguntas orientadoras propuestas. Así mismo, y para los cursos más pequeños, se realizaron lecturas relacionadas con distintos valores a partir de las cuales los niños desarrollaron talleres y una serie de dibujos que expresaban la introyección de los temas abordados.

\section{Discusión}

El proceso de investigación, orientado al fortalecimiento de las habilidades y competencias a nivel de convivencia y cultura ciudadana en la población estudiantil, permitió involucrar diferentes actores como lo son padres de familia, maestros y estudiantes, los cuales contribuyeron mediante trabajo colaborativo a materializar actividades que conducen a experiencias significativas, como lo fueron el desarrollo de huertos escolares y familiares, en las cuales los estudiantes realizaron diferentes tipos de actividades que iniciaron a partir del aula de clases desde los procesos de indagación y consultas, y que les permitieron construir conceptos y aprendizajes en relación al tema de interés investigativo, el cual, mediante su puesta en marcha desde el componente experimental, facilitó la exposición a eventos que requieren la práctica de una buena convivencia.

Por otro lado, las diferentes actividades asociadas a estrategias pedagógicas para el fortalecimiento de las habilidades a nivel de convivencia ciudadana, que derivan de la reflexión de los videos, talleres y mesas de trabajo desarrolladas por la población de estudio, se realizaron en el marco de la IEP basado en el uso de las TIC, teniendo como fin promover un aprendizaje colaborativo, contextualizado y problematizado, en el que los estudiantes tuviesen una participación activa. Las estrategias didácticas y el uso de las TIC como parte de la enseñanza y el aprendizaje, permiten la motivación, mejorando el desarrollo de la competencia lectora (Villa, 2013).

Así mismo, se obtuvieron evidencias escritas en las que el estudiante logró realizar una reflexión desde las preguntas orientadoras, lo que muestra que poseen amplias habilidades para reconocer sus errores, debilidades y fortalezas, por lo que pueden reconocer que ciertas actitudes y o comportamientos inapropiados o que atentan contra los derechos de sí mismos y los del prójimo, son el reflejo de aspectos intrínsecos de la complejidad social, y en gran medida de lo relativo a la disfuncionalidad familiar, convirtiendo a la escuela en un lugar de rehabilitación ciudadana para quienes se están formando.

Teniendo en cuenta los resultados obtenidos con los estudiantes de la Institución Educativa Agropecuaria Otilia Mena Álvarez, en el marco de este estudio de tipo cualitativo propositivo, se considera importante continuar replicando este tipo de prácticas a partir de la IEP, que generen espacios de desarrollo de estrategias para el mejoramiento y fortalecimiento de los procesos de convivencia escolar y cultura ciudadana, haciendo uso de las TIC como herramientas facilitadoras en los procesos de aprendizaje. Se recomienda también, la incorporación de estrategias que estén relacionadas con diagnósticos y proyectos de interés para la institución y la comunidad. Con respecto a la tecnología a futuro conviene investigar sobre alfabetización digital de las comunidades. 


\section{Referencias}

Alfaro, A., Peralta, G., Hurtado, J. y Contreras, Y. (2014). La convivencia y la mediación de conflictos como estrategia pedagógica en la vida escolar. Revista Panorama Económico, (22), 169-190.

Belalcazar, C. y Molina, M. (2017). Socialización de experiencias educativas mediadas por TIC en pro de la convivencia y cultura de paz. [Tesis de Maestría]. Centro de tecnologías de la academia, Universidad de la Sabana.

Bolívar, A. (2004). Ciudadanía y escuela pública en el contexto de diversidad cultural. Revista Mexicana de investigación Educativa, 9(20), 15-38.

Colciencias (2011). Las Comunidades de Aprendizaje, Prácticas, Saber, Conocimiento y Trasformación Apoyadas en las Nuevas Tecnologías de la Información y la Comunicación. Bogotá D.C.: Colciencias.

Garrell, T. (2009). Aprender a convivir. Caracas: Laboratorio Educativo.

Hernández, J., Alvarado, L. y Velazco, J. (2013). Participación ciudadana, tecnologías de información-comunicación y opinión pública. TEACS, 5(11). 25-33.

Illera, M. (2005) Convivencia y cultura ciudadana: Dos pilares fundamentales del derecho policivo. Revista de Derecho. (23), 240-259.

Marrugo, G., Gutiérrez, J., Concepción, I. y Concepción, M. (2016). Estrategia de Convivencia Escolar Para la Formación de Jóvenes Mediadores de Conflictos. Escenarios, 14(1). 72-84

Megías, M. (2011). La convivencia escolar y cómo resolver los conflictos y la violencia en el aula. Granada: ADICE.

Mejía, M. y Manjarrez, M. (2010). La investigación como estrategia pedagógica: Una apuesta por construir pedagogías críticas en el siglo XXI. Bogotá, D.C.: Programas ONDAS- Colciencias.
Mena, I., Romagnoli, C. y Valdés, A. (2006). Convivencia escolar. Documento Valoras UC. Recuperado de http://ww2.educarchile.cl/UserFiles/P0001/File/ convivencia_escolar.pdf

Ramírez, C. (2010). Las TICS en el Aula. Granada: NNTT.

República de Colombia. Ministerio de Educación Nacional. 2006. Plan Nacional Decenal de Educación PNDE 2006 2016. Bogotá, D.C.: Mineducación. Disponible en http://www.mineducacion.gov.co/1621/articles-312490_archivo_pdf_plan__decenal.pdf. Consultado el 15.07.2015

Romero, K. y Contreras, E. (2015). Revisión teórica sobre el post-conflicto: una oportunidad para empoderar a mujeres víctimas de desplazamiento. Cultura Educación y Sociedad, 6(1). Recuperado de https://revistascientificas.cuc. edu.co/culturaeducacionysociedad/article/view/757

Ruiz-Silva, A. y Choux, E. (2005). La formación de competencias ciudadanas. Bogotá, D.C.: Asociación Colombiana de Facultades de Educación ASCOFADE.

Samper, J. y Maussa, E. (2014). Desarrollo moral y competencias ciudadanas en la juventud universitaria. Jurídicas CUC, 10(1), 43 - 60.

Valenzuela, G. y Rochin, L. (2013). Libertad de expresión y su impacto en la democracia. Jurídicas CUC, 9(1), 89 100.

Villa, I. (2013). Competencia Lectora y el Uso de las Nuevas Tecnologías de la Información y Comunicación. Reading competition and the use of new technologies of information, 11(1), 7-22. 